\title{
Data-driven algorithm to classify the degree of isotropy in the bidirectional reflectance distribution function
}

\author{
Anne W. Werkley,* Samuel D. Butler, Todd V. Small, \\ and Michael A. Marciniak \\ Air Force Institute of Technology, Department of Engineering Physics, \\ Wright-Patterson Air Force Base, Ohio, United States
}

\begin{abstract}
The bidirectional reflectance distribution function (BRDF) is used to describe reflectances of materials by calculating the ratio of the reflected radiance to the incident irradiance. While it was found that the isotropic models maintained symmetry about $\phi_{s}=\pi$, such symmetry was not maintained about the $\theta_{s}=\theta_{i}$ axis, except for close to the specular peak. This led to the development of a data-driven metric for how isotropic a BRDF measurement is. Research efforts centered around developing an algorithm that could determine material anisotropy without having to fit to models. This algorithm was tested using high fidelity data (containing off-axis BRDFs), which was collected via a modified Complete Angle Scatter Instrument (CASI ${ }^{\circledR}$ ) with a CCD array detector. The algorithm accurately characterized the degree of isotropy for four out of five materials and worked for cases where the BRDF is higher than $100 \mathrm{sr}^{-1}$. This algorithm is intended to improve BRDF characterization, and the applications of light curve analysis, scene generation, and remote sensing. (C) The Authors. Published by SPIE under a Creative Commons Attribution 4.0 International License. Distribution or reproduction of this work in whole or in part requires full attribution of the original publication, including its DOI. [DOI: 10.1117/1.OE.60.9.094108]
\end{abstract}

Keywords: bidirectional reflectance distribution function; optical scatter; microfacet; remote sensing; computer vision; scene generation.

Paper 20210542 received May 23, 2021; accepted for publication Sep. 2, 2021; published online Sep. 20, 2021

\section{Background}

The bidirectional reflectance distribution function (BRDF) is a description of the ratio of the reflected radiance to the incident irradiance, ${ }^{1}$

$$
f_{r}\left(\hat{\omega}_{i}, \hat{\omega}_{s}, \lambda\right)=\frac{d L_{r}\left(\hat{\omega}_{i}, \hat{\omega}_{s}, \lambda\right)}{d E_{i}\left(\hat{\omega}_{i}, \lambda\right)}
$$

where $f_{r}$ is the BRDF, $\hat{\omega}_{i}$ is the incident vector with respect to the material's surface normal, $\hat{\omega}_{s}$ is the "scattered" vector, $L_{r}$ is the reflected radiance, $E_{i}$ is the incident irradiance, and $\lambda$ is the respective wavelength. Wavelength was not considered in this study, as only one wavelength, $\lambda=632.8 \mathrm{~nm}$, was used to illuminate samples.

The rendering equation at an intersection point with a material, which is where the BRDF often arises in practical applications, is ${ }^{2}$

$$
L_{s}\left(\hat{\omega}_{s}\right)=L_{e}\left(\hat{\omega}_{s}\right)+\int_{\Omega_{+}} f_{r}\left(\hat{\omega}_{i}, \hat{\omega}_{s}\right) L_{i}\left(\hat{\omega}_{i}\right) \cos \theta_{i} \mathrm{~d} \hat{\omega}_{i},
$$

where $L_{s}$ is the scattered radiance, $L_{i}$ is the incident radiance, $L_{e}$ is the emitted radiance, and $\Omega_{+}$represents the incident hemisphere. This equation shows that the BRDF is crucial to describe the reflected radiance in applications such as scene generation and remote sensing.

BRDF is commonly represented using either physical optics models or microfacet models. The microfacet models use a geometric optics approximation to reduce computational complexity, making modeling BRDF more feasible for most applications, including light curve analysis ${ }^{3}$

*Address all correspondence to Anne W. Werkley, awerkley@gmail.com 
and scene generation. ${ }^{4}$ These models approximate a rough surface as a set of microfacets from which incident radiation is scattered. ${ }^{4}$

Butler composed an equation describing the general form of the microfacet model, in terms of standardized Rusinkiewicz coordinates ${ }^{5}$ and a uniform notation ${ }^{6}$ in Eq. (3). ${ }^{1}$ Generally, microfacet models include a surface reflection term, which constitutes the first term in Eq. (3), and occasionally a volumetric scattering term (the second term), and almost always a Lambertian scattering term, (the final term), ${ }^{6}$

$$
f_{r}\left(w_{i}, w_{o}\right)=\rho_{s} P\left(\hat{\omega}_{i}, \omega_{s}\right) D\left(\hat{\omega}_{h}\right) F\left(\theta_{d}\right) G\left(\hat{\omega}_{i}, \hat{\omega}_{s}\right) \sigma\left(\theta_{i}, \theta_{s}\right)+\rho_{v} V\left(\hat{\omega}_{i}, \hat{\omega}_{s}\right)+\frac{\rho_{d}}{\pi} .
$$

Breaking down each component of Eq. (3) further, $G$ is the geometric attenuation factor and describes the shadowing and masking of reflections off one facet by another facet. ${ }^{6} F\left(\theta_{d}\right)$ is the Fresnel reflectance and can be replaced by a Rayleigh-Rice Polarization factor term in various BRDF models. ${ }^{7} \sigma\left(\theta_{i}, \theta_{s}\right)$ is a term used to convert from scattering cross section to BRDF. ${ }^{6}$ $D\left(\hat{\omega}_{h}\right)$ is the distribution function of the microfacet surface normals, where $\hat{\omega}_{h}$ is used to refer to these surface normals. $P$ merely represents a prefactor term included for some of the microfacet models to get each in the same form. ${ }^{6}$ The distribution function was the main focus of this study, as it is the term in the overall BRDF equation that changes between isotropic and anisotropic models.

There exists a whole host of microfacet models employing various distribution functions, some employing isotropic distributions and others employing anisotropic distributions. For the context of this paper, anisotropy is given to mean any surface that produces directionally dependent reflection (as the specular term in microfacet models describes surface reflection). This can be due either to a material with directionally dependent surface characteristics, such as machine linings or a change in material across a surface (an inhomogeneous sample). Equations (4)-(6) describe some of the distribution functions employed in microfacet models. Equation (4) shows the Beckmann distribution function (a Gaussian distribution), controlled by width $\sigma_{g}$, and the in-plane direction of the microfacet normal, $\theta_{h}$. This distribution function (or an approximation where $\tan \left(\theta_{h}\right)=\theta_{h}$ ) results in isotropic reflection and is used in the Ward-Duer, ${ }^{8}$ Torrance-Sparrow, ${ }^{4}$ Cook-Torrance, ${ }^{9}$ and Priest microfacet models. ${ }^{10}$

$$
D_{g}\left(\theta_{h}\right)=\frac{1}{2 \pi \sigma_{g}^{2} \cos ^{4}\left(\theta_{h}\right)} \exp \left[-\left(\frac{\tan ^{2}\left(\theta_{h}\right)}{2 \sigma_{g}^{2}}\right)\right]
$$

The Ward-Duer model ${ }^{8}$ can be modified to use an anisotropic distribution function in lieu of an isotropic function. This anisotropic distribution is shown in Eq. (5). It is worth noting that to get Eq. (5) in the form of isotropic models, one can set $m_{x}=m_{y}=m=\sqrt{2} \sigma_{g}$. However, in anisotropic models, $m_{x}$ and $m_{y}$ are allowed to vary along azimuthal planes. Another difference between models is the required inclusion of the out-of-plane azimuthal coordinate of the microfacet surface normal, $\phi_{h}$.

$$
D\left(\hat{\omega}_{h}\right)=\frac{1}{\pi m_{x} m_{y} \cos ^{4} \theta_{h}} \exp \left[-\tan ^{2} \theta_{h}\left(\frac{\cos ^{2} \phi_{h}}{m_{x}^{2}}+\frac{\sin ^{2} \phi_{h}}{m_{y}^{2}}\right)\right]
$$

Not all models employ a Gaussian distribution function to describe the orientation of microfacets. Equation (6) is a Hyper-Cauchy distribution function, where $q$ and $s$ are parameters used to control the width of the distribution function. When $q=3 / 2$, a function similar to a Lorentzian results, and a Gaussian-like function results when $q$ is large. ${ }^{6}$ The Hyper-Cauchy distribution function is used in the Wellems microfacet model. ${ }^{6,11}$

$$
D_{g}\left(\theta_{h}\right)=\frac{(q-1)(s \sqrt{2})^{2 q-2}}{\pi \cos ^{4}\left(\theta_{h}\right)\left((s \sqrt{2})^{2}+\tan ^{2}\left(\theta_{h}\right)\right)^{q}}
$$

Beyond the models enumerated above, there also exists a cosine-lobe distribution function, which can describe either isotropic and anisotropic reflection. Cosine-lobe distribution functions 
are employed in the Blinn-Phong ${ }^{6,12}$ (isotropic only) and the Ashikhman-Shirley (both isotropic and anisotropic) models. ${ }^{6,13}$

As one can observe through the host of equations enumerated and those not listed, there is no standard or agreed upon BRDF model to describe isotropy or anisotropy. ${ }^{6,14}$ The majority of these models solely employ an isotropic distribution function, ${ }^{6}$ which can provide problematic fits, especially for more specular/glossy BRDFs. This study considers the degree of isotropy presented by various samples and develops a metric to assess how not isotropic a material is.

\section{Methodology}

As mentioned previously, the distribution function changes significantly between isotropic and anisotropic materials. For many materials, the distribution function is the most significant term. Thus, the distribution function was the first investigation of this study to determine what constitutes "isotropic" reflection. While several distribution functions were listed in the previous section, the Beckmann distribution function [Eq. (4)] was used as it is relatively computationally inexpensive and commonly employed.

As Eq. (4) depends on $\theta_{h}$, the impact of changing $\theta_{i}, \theta_{s}$, and $\phi_{s}$ on $\theta_{h}$ was investigated. In plotting the distribution function at varying $\theta_{i}$ and $\sigma_{g}$, the contour plot showed that the peak of the distribution function occurs at $\theta_{i}=\theta_{s}$ and $\phi_{s}=\pi$, which is the specular peak, when $\phi_{i}=$ $0 \mathrm{deg}$ (representing the forward scatter case). The smaller $\sigma_{g}$ was made to be, the narrower the peak. These results were to be expected, as $\theta_{h}=0$ deg is the peak of the distribution function, and only occurs where $\theta_{i}=\theta_{s}$ and $\phi_{s}=\pi$.

Considering these characteristics of the distribution function, the symmetry of the distribution function about the specular peak was then evaluated to develop a definition of isotropy. Symmetry was first evaluated about the point $\phi_{s}=\pi$. First, an arbitrary $\theta_{i}$ and $\theta_{s}$ were set and plugged into the distribution function [Eq. (4)]. The relationship in Eq. (7) was then used to show that the distribution function is symmetric about $\phi_{s}=\pi$, as there is no difference if one moves a distance $b$ (arbitrary) in either direction from this point.

$$
D\left(\theta_{i}, \theta_{s}, \pi+b\right)-D\left(\theta_{i}, \theta_{s}, \pi-b\right)=0
$$

The same analytic analysis was done moving an arbitrary distance, $b$, in $\theta_{s}$ space. For small increments of $\theta_{s}$ off of $\theta_{i}=\theta_{s}(0.5 \mathrm{deg})$, the relative percentage differences between the distribution functions were small (difference of $0.2 \%$ ), yet significant in comparison to the zero difference in $\phi_{s}$. The asymmetry in $\theta_{s}$ is exacerbated as $\sigma_{g}$ decreases, as the relative difference for $\sigma_{g}=0.01$ is $1.5 \%$ in comparison to the $0.2 \%$ for $\sigma_{g}=0.1$. The asymmetry in $\theta_{s}$ was also generalized by finding an analytic expression, as in Eq. (7) and then by taking the Taylor series expansion about $\theta_{h}=0$, (i.e., the specular peak). The analytical expression for the symmetry about $\theta_{i}=\theta_{s}$ is reduced to,

$$
\begin{aligned}
D\left(\theta_{i}, \theta_{i}+b, \phi_{s}\right)-D\left(\theta_{i}, \theta_{i}-b, \phi_{s}\right)= & \frac{8}{\pi} \frac{-1}{\cos \left(b-\theta_{i}\right)+\cos ^{4}\left(\theta_{i}\right)} \\
& \exp \left[\frac{-2-\cos \left(b+\phi_{s}\right)+\ldots}{8 \cos \left(b-\theta_{i}\right)+\cos ^{4}\left(\theta_{i}\right)}\right]+\ldots
\end{aligned}
$$

Equation (8) does not reduce to zero, unlike with Eq. (7), indicating that symmetry is not maintained about $\theta_{i}=\theta_{s}$. For the Taylor series expansion about $\theta_{h}=0$, the expression for the distribution function reduced to,

$$
D\left(\theta_{h}\right)=\frac{1}{2 \pi \sigma_{g}^{2}}+\frac{\theta_{h}^{2}\left(-1+4 \sigma_{g}^{2}\right)}{4 \pi \sigma_{g}^{4}} .
$$

In the case that $\theta_{h}$ is near zero, the expression reduces to the first term of Eq. (9), which is why the degree of symmetry (i.e., smaller relative percentage difference) in $\theta$ is inversely proportional to $\sigma_{g}$. 


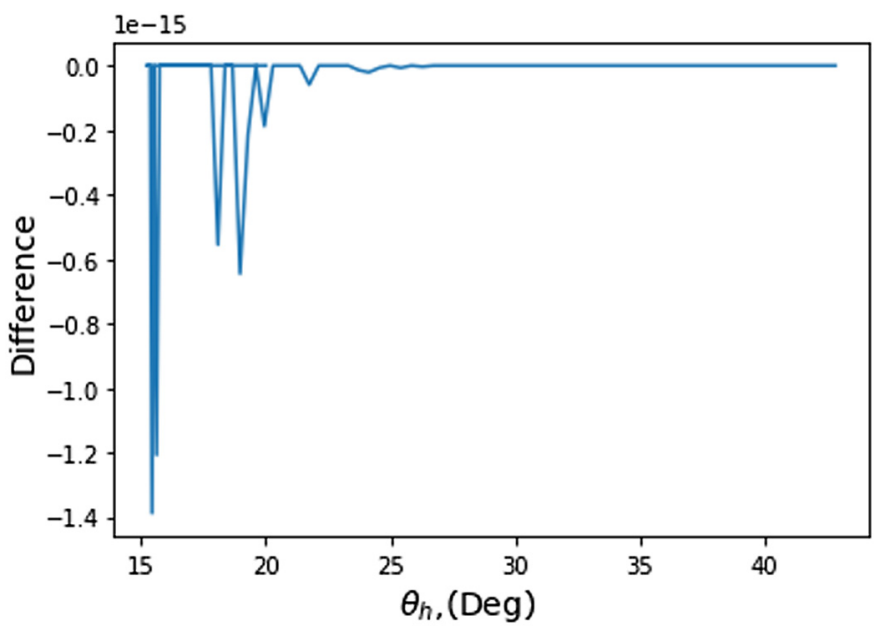

Fig. 1 Difference between the full BRDF (un-normalized) functions when $\theta_{i}$ and $\sigma_{g}$ are kept constant at $40 \mathrm{deg}$ and $0.1 \mathrm{deg}$, respectively and $\phi_{s}$ is varied from $3 \pi / 4$ to $5 \pi / 4$ (in radians). As one can see, the difference is minimal, on the order of $10^{-15}$, which is likely due to computational rounding error in the trigonometric functions.

These relationships were then expanded to the full BRDF, using a simplification of Eq. (3), and the Rayleigh-Rice Polarization factor, $Q$, in place of $F$. An analytic evaluation was difficult to do in the case of the full BRDF, so the differences are shown in Fig. 1 for two $\phi_{s}$ values that are equidistant from $\phi_{s}=\pi$, specifically $\phi_{s}=3 \pi / 4$ and $5 \pi / 4$. In Fig. 1 , the plot is in terms of $\theta_{h}$, as the constant $\theta_{i}$ and varying $\phi_{s}$ values were plugged into $\theta_{h}$ before solving for the BRDF (which is a function of $\theta_{h}$ ). These differences are on the order of $1 \times 10^{-15}$, which is likely due to computational rounding errors. Thus, it is safe to say that symmetry in $\phi_{s}$ is maintained with the full BRDF, despite the additional terms.

It is worth noting that although the analysis of isotropic BRDF models was done for microfacet models only (and simulated data were generated using the aforementioned models), some elements of physical optics models were used in this study. Specifically, BRDFs were converted to direction cosine space, as the isotropic Beckmann distribution function [Eq. (4)] is symmetric in this space. This distrbution function can be correlated to the direction cosine space coordinates $^{15}$ as shown by Eq. (10), where $\Delta \alpha$ and $\Delta \beta$ represent the direction cosine space coordinates. $^{16}$

$$
\tan ^{2}\left(\theta_{h}\right)=\frac{\Delta \alpha^{2}+\Delta \beta^{2}}{\cos \left(\theta_{i}\right)+\cos \left(\theta_{s}\right)}
$$

This symmetry is further demonstrated analytically in Eqs. (11) and (12), where $\Delta \alpha$ and $\Delta \beta$ are plugged into Eq. (4) to evaluate the rotational symmetry. In Eqs. (11) and (12), b is given to represent a constant step in direction cosine space.

$$
\begin{aligned}
& D(\Delta \alpha+b, \Delta \beta)-D(\Delta \alpha-b, \Delta \beta)=0 \\
& D(\Delta \alpha, \Delta \beta+b)-D(\Delta \alpha, \Delta \beta-b)=0
\end{aligned}
$$

The symmetry of isotropic BRDFs about $\phi_{s}=\pi$ and in direction cosine space were useful parameters in developing an algorithm assessing the degree of isotropy. (This algorithm will be detailed in the next section.) However, it should be noted that anisotropic models were also investigated but did not produce any fruitful metric for assessing a material's anisotropy. This, paired with the fact that anisotropic models rely heavily on sample alignment (so that $\phi_{i}$ produces a $\phi_{h}=0$ ) is why the focus of this study is on the degree of isotropy and not the degree of anisotropy. 


\section{Results}

\subsection{Algorithm}

The algorithm, used to assess the degree of isotropy present in simulated and exprimental data, is detailed below. Given BRDF measurements of a material at a certain $\theta_{i}$ in scatter coordinate space:

1. Measure the mean symmetry about $\phi_{s}=\pi$.

2. Convert to direction cosine space.

3. Measure the diameter, $x$ and $y$ center of each contour, the full width half maximum (FWHM) of the data against $\Delta \alpha$, and the eccentricity of each contour centered about the peak BRDF value.

4. Scale the FWHM and diameter measurements according to $\theta_{i}$.

5. Combine all of the data for the material (for all $\theta_{i}$ ), and find the standard deviations for scaled diameter, $(x, y)$ center, FWHM, and eccentricity.

6. Find the mean of the symmetry and eccentricity measurements.

7. Compare each of these parameters to the baseline (find percentage differences).

8. Weight each parameter by the appropriate factor.

9. Find the final score and categorize each material as isotropic or anisotropic. Scores lower than 1 are highly isotropic, scores $1-10$ are materials tha lean isotropic, scores greater than 10 are anisotropic, and those $>30$ are highly anisotropic.

\subsection{Simulated Data/Beam Signature Data}

The algorithm developed from the investigation of BRDF models was first tested on simulated data sets to establish baselines for isotropy. These model-generated datasets served as references and were to used established an overall algorithm, as well as a baseline uncertainty for the algorithm. First, the mean symmetry was found by folding the data in half about the point $\phi_{s}=\pi$ and finding the mean difference above and below that point. The mean difference for the symmetry was found to be on the order of computational rounding error. Next, the data were converted to direction cosine space, and the FWHM was found by plotting the BRDF data against $\Delta \alpha$. This was done because although the distribution function is symmetric in direction cosine space, the $\mathrm{BRDF}$ is not fully symmetric, yet the FWHM remains constant as $\theta_{i}$ increases. Figure 2 shows a contour plot of the simulated BRDF data in direction cosine space. This contour plot was used to measure several parameters of the algorithm, including the scaled diameter, $(x, y)$ center, and eccentricity.

Referring to Fig. 2, each contour is measured by converting the image to pixel space and performing a Hough transform of the image to measure various parameters. For example, the scaled diameter parameter measures the diameter of the outermost contour, multiplied by a scaling factor to account for how a change in $\theta_{s}$ is not the same for each $\theta_{i}$ in $\Delta \beta$. The outermost contour level is set to be two orders of magnitude below the peak. The eccentricity parameter measured the eccentricity of each contour, and recorded a mean and standard deviation at each $\theta_{i}$. The $(x, y)$ center parameter measures the standard deviation of where each contour is centered, to rule out anisotropic cases where the eccentricity remained consistent, but the contours were measured from rapidly varying points. Table 1 summarizes the results of these measured parameters for the simulated isotropic data for $\sigma_{g}=0.005$. The symmetry parameter is not recorded in the table, but is recorded as 0, meaning that there is no difference as one moves off $\phi_{s}=\pi$ in either direction. Several of the columns in Table 1 are measured in pixels, as these measurements are from the Hough transform of contour plots (similar to Fig. 2) and thus recorded in image space rather than scatter coordinate space. Since the scale of these plots are set to the same range in direction cosine space, the parameter measurements in pixel space are consistent.

Before testing the algorithm on experimental data, the algorithm was also run on beam signature data. The data collection set-up is described fully in Small's SPIE conference paper. ${ }^{17,18}$ Overall, the relative uncertainty present in data collected via the modified Complete Angle 


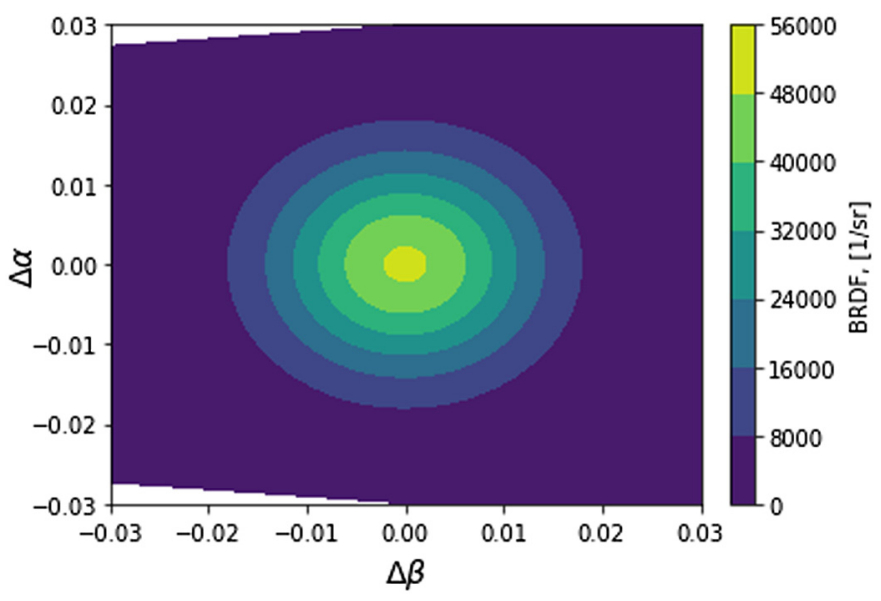

Fig. 2 Simulated isotropic BRDF data for $\sigma_{g}=0.005$ and $\theta_{i}=20^{\circ}$. The average eccentricity was $0.897 \pm 0.006$ for this particular $\theta_{i}$, indicating that the eccentricity of contours remains relatively low (near circular) as well as constant for isotropic BRDFs.

Table 1 Table summarizing various parameters for the simulated isotropic data collected at $\theta_{i}=20 \mathrm{deg}, 40 \mathrm{deg}$, and $60 \mathrm{deg}$ for a set $\sigma_{g}=0.005$. The standard deviations for each parameter are at most $16 \%$ of the mean, which indicates that for isotropic materials, the measured parameters shift little as $\theta_{i}$ increases.

\begin{tabular}{lcccccc}
\hline \hline Material & $\begin{array}{c}\theta_{i} \\
(\mathrm{deg})\end{array}$ & $\begin{array}{c}\text { Mean } \\
\text { eccentricity }\end{array}$ & $\begin{array}{c}\text { Scaled } \\
\text { diameter } \\
\text { (pixels) }\end{array}$ & $\begin{array}{c}X \text { center } \\
\text { (pixels) }\end{array}$ & $\begin{array}{c}Y \text { center } \\
\text { (pixels) }\end{array}$ & $\begin{array}{c}\text { FWHM } \\
\text { (unitless) }\end{array}$ \\
\hline Sim isotropic & 20 & 0.89 & 130.95 & 132.98 & 114.34 & 0.012 \\
Sim isotropic & 40 & 0.81 & 136.41 & 136.63 & 109.03 & 0.014 \\
Sim isotropic & 60 & 0.82 & 136.41 & 136.63 & 108.50 & 0.011 \\
Average & - & 0.84 & 134.80 & 134.79 & 110.62 & 0.012 \\
STD & - & 0.04 & 3.35 & 1.82 & 3.23 & 0.002 \\
\hline \hline
\end{tabular}

Scatter Instrument (CASI) system was between $4 \%$ and $17 \%$, with the highest uncertainty being present in the more diffuse samples due to the neutral density filter uncertainty, which affects the CCD uniformly. ${ }^{19}$ The beam signature was a Helium-Neon laser source passing directly to the CCD detector. As the beam did not reflect off anything, its radial symmetry was assumed to be representative of an isotropic sample. Any asymmetries captured are likely due to noise or from the off-axis parabolic mirror in the laser set-up. There exist aberrations and imperfections in the optical set-up, so the beam is not perfectly Gaussian at the detector. Figure 3 shows the beam signature with a Gaussian filter of $\sigma=1$ to filter out some of the noise.

The beam signature data compare well to the simulated data set, as shown in Table 2 . However, measurements were only taken for $\theta_{i}=0 \mathrm{deg}$, as the beam does not reflect off any surfaces. The differences between the beam signature and simulated data helped to establish baselines because the beam was not perfectly symmetric compared to the simulated data. The baselines are summarized in Table 3.

The scaled diameter and FWHM baselines were set by the simulated data set, as these change with $\theta_{i}$, and the beam signature data only has one $\theta_{i}$, whereas three $\theta_{i}$ 's were recorded for the simulated data. The standard deviation of the $(x, y)$ centers (referring to the centers of contours) is also pulled from the simulated data, as it was actually higher for this data set than the beam signature. The mean eccentricity and standard deviation of eccentricity (which are pulled from the beam signature data) are thus used to account for noise in the experimental setup by serving as isotropy baselines. Using these baselines, a total relative uncertainty in the algorithm was 


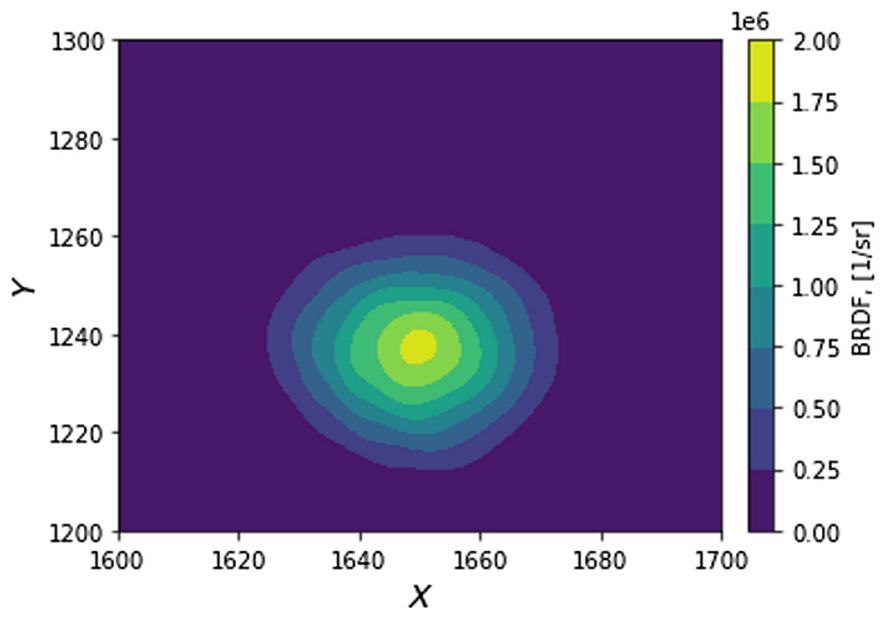

Fig. 3 Contour plot of the beam signature in pixel coordinate space. A Gaussian filter with a $\sigma$ of 1 was applied to this image.

Table 2 Table summarizing the parameters measured for the beam signature. These measurements have been reported to help determine a baseline error in the data collected and the algorithm used to fit the data. The standard deviation represents the uncertainty in these measurements. Based upon the developed methodology, the beam signature is classified as "solidly isotropic."

\begin{tabular}{lcccccc}
\hline \hline Material & $\begin{array}{c}\text { Mean dif } \\
\text { symm (\%) }\end{array}$ & $\begin{array}{c}\text { Min dif } \\
\text { symm (\%) }\end{array}$ & $\begin{array}{c}X \text { center } \\
\text { (pixels) }\end{array}$ & $\begin{array}{c}Y \text { center } \\
\text { (pixels) }\end{array}$ & Eccentricity & Class \\
\hline Beam sig & 9.11 & $6.90 \times 10^{-5}$ & $166.65 \pm 1.00$ & $53.86 \pm 2.25$ & $0.73 \pm 0.04$ & Solidly isotropic \\
\hline \hline
\end{tabular}

Table 3 Table summarizing the various baselines and weights for each parameter of isotropy. STD stands for standard deviation.

\begin{tabular}{lccccc}
\hline \hline Baseline & $\begin{array}{c}\text { Scaled diameter } \\
\text { (STD) }\end{array}$ & $\begin{array}{c}X / Y \text { center } \\
\text { (STD) }\end{array}$ & $\begin{array}{c}\text { FWHM } \\
\text { (STD) }\end{array}$ & $\begin{array}{c}\text { Eccentricity } \\
\text { (value + STD) }\end{array}$ & $\begin{array}{c}\text { Mean } \\
\text { (\% difference) }\end{array}$ \\
\hline Value & 3.35 & 3.23 & 0.002 & $0.73 \pm 0.04$ & 9.11 \\
Weight & 0.05 & 0.18 and 0.18 & 0.05 & $0.18 \& 0.18$ & 0.18 \\
\hline \hline
\end{tabular}

determined. The numeric value for degree of isotropy was computed as $\sum\left(w_{i} x_{i}\right)$, where $w_{i}$ is the weight, and $x_{i}$ is each individual metric value. Using the weights of each parameter paired with the relative uncertainty (standard deviation divided by the average value), error propagation was performed to find a total relative uncertainty of $1.64 \%$.

\subsection{Testing on Experimental Data}

After analyzing the simulated data and beam signature to establish isotropy baselines, five different samples were used to evaluate the algorithm. The first sample was a highly specular metallic front-surface reflecting laboratory mirror ("lab mirror" sample) with no visible scratches or machine linings. The second sample was a Kapton sample, a semi-transparent film, which was less reflective than the mirror sample. The third sample was a "polished aluminum" sample with visible circular machine lines; the sample was highly reflective (though less so than the lab mirror sample). The fourth sample was a solar panel consisting of a clear top layer and layer beneath lined with conductive grid lines, measuring approximately $1-\mathrm{mm}$ wide with equivalent spacing in between. The final sample was a "metal mesh," a 2D grid that was highly 
Table 4 Table summarizing each of the materials and the predictions for each material.

\begin{tabular}{lc}
\hline \hline Material & Prediction \\
\hline Lab mirror & Isotropic \\
Kapton & Leans isotropic \\
Polished aluminum & Anisotropic \\
Solar panel & Highly anisotropic \\
Metal mesh & Anisotropic \\
\hline \hline
\end{tabular}

transmissive. As the samples were all illuminated in the visible, visual inspection was done on each of the samples to predict the degree of isotropy. Table 4 lists each of the materials and the predictions for each.

Each of the five samples was analyzed by employing the algorithm enumerated in Sec. 3.1. Figure 4 shows a plot of the lab mirror sample in direction cosine space. The peak BRDF of this material was on the order of $10^{6} \mathrm{sr}^{-1}$, indicating a highly specular material. The lab mirror compares similarly eccentricity-wise to the simulated isotropic and beam signature data. As a note, the BRDF data in Fig. 4 has been normalized by the maximum value, and the contours only include BRDF data two orders of magnitude below the peak.

Figure 4 shows the $20 \mathrm{deg}, 40 \mathrm{deg}$, and $60 \mathrm{deg}$ measurements in direction cosine space. The results of algorithm on this sample are summarized in Table 5. The lab mirror compares well to the baselines. When scaled, the FWHM is the most consistent out of all of the measured materials with a standard deviation of 0.005 , just slightly higher than the baseline of 0.002 . The $(x, y)$ center coordinates also have very low standard deviations (the standard deviations are actually both lower than the baseline standard deviation of 3.23 pixels), which points further toward isotropy. These $(x, y)$ centers are measurements of the centers of each contour measured for each $\theta_{i}$ contour plot in direction cosine space. The mean overall eccentricity is higher than the baseline of 0.73, although the standard deviation is somewhat higher than the baseline of 0.04 . However, for each $\theta_{i}$, the standard deviation remained consistent at 0.01 for the eccentricity, which strongly indicates isotropy. The only point against this material in terms of isotropy is the scaled diameter column, which deviated from the baseline significantly. This metric is possibly problematic for two reasons. First, there may be issues aligning the sample properly, where the reflected beam could be elongated as $\theta_{i}$ increases, causing the diameter to vary more than expected. Second, the outer contour measurement relies upon the contours set by Python, which vary somewhat as BRDF increases as $\theta_{i}$ increases. As the data are narrowed down to the peak BRDF plus two orders of magnitude below, the cutoff for the outer contour changes with $\theta_{i}$, possibly accounting for the higher standard deviation for the scaled diameter. Otherwise, the algorithm identified the material as isotropic, matching up with the previous expectation that a smooth surface with no visible scratches or marks should reflect light isotropically.
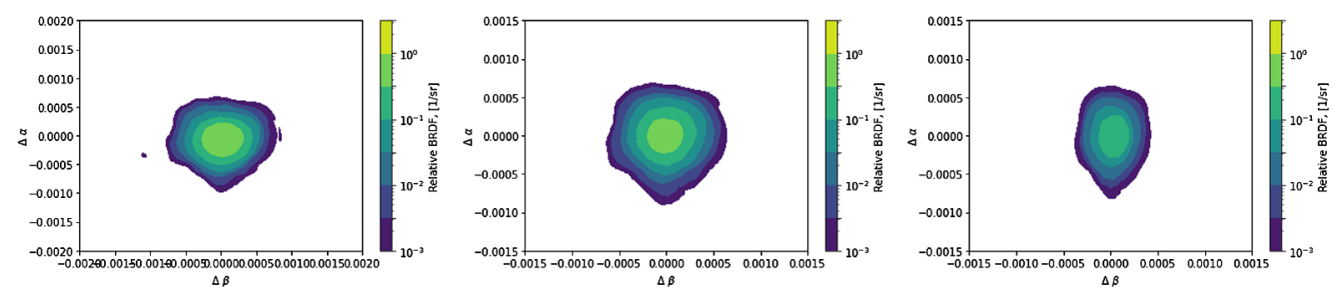

Fig. 4 Lab mirror in direction cosine space. The plot occupies a smaller region in this space as $\theta_{i}$ increases, becoming less symmetric. Overall, the contours appear relatively symmetric (in comparison to the simulated data and beam signature data) for each of the plots, maintaining near circular eccentricities. From left to right, the plots are $20 \mathrm{deg}, 40 \mathrm{deg}$, and $60 \mathrm{deg}$, respectively. 
Table 5 The lab mirror overall rates as isotropic. This is because the standard deviations reported for the FWHM, eccentricity, and $(x, y)$ centers compare well to the baselines set by beam signature and simulated isotropic data. The symmetry is also high here as it deviates little in symmetry from the beam signature data, which is the baseline. Ecc. stands for eccentricity.

\begin{tabular}{lccccccc}
\hline \hline Material & $\begin{array}{c}\text { Mean } \\
\text { symmetry } \\
\text { (\% dif) }\end{array}$ & $\begin{array}{c}\text { Scaled } \\
\text { diameter } \\
\text { (pixels) }\end{array}$ & $\begin{array}{c}\text { Scaled } \\
\text { FWHM } \\
\text { (unitless) }\end{array}$ & $\begin{array}{c}X \text { center } \\
\text { (pixels) }\end{array}$ & $\begin{array}{c}Y \text { center } \\
\text { (pixels) }\end{array}$ & Ecc. & Class \\
\hline Lab mirror & 9.39 & 44.27 & 0.016 & 134.42 & 110.12 & 0.81 & Isotropic \\
& \pm 1.60 & \pm 17.18 & \pm 0.005 & \pm 1.51 & \pm 1.60 & \pm 0.10 & \\
\hline \hline
\end{tabular}
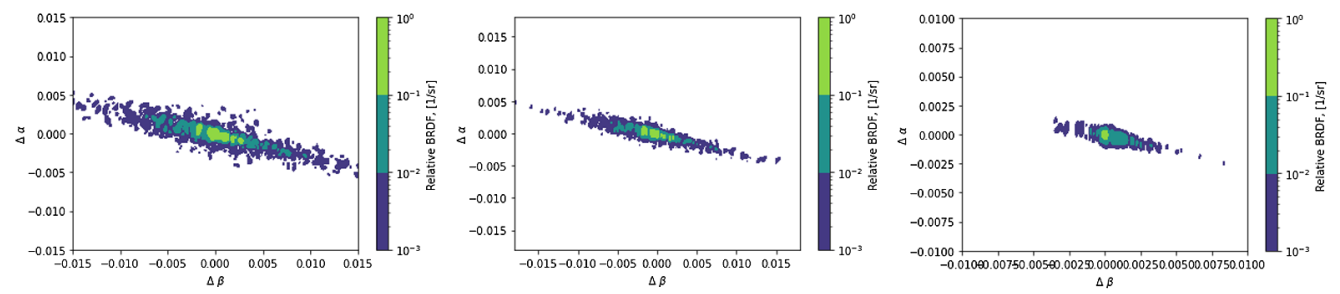

Fig. 5 Plot showing the polished aluminum sample in direction cosine space. The sample spans a wider range in direction cosine space than the lab mirror sample and is stretched in $\Delta \beta$ compared to $\Delta \alpha$, indicating material anisotropy. The reflected beam also shrinks in size as $\theta_{i}$ increases, similar to the lab mirror sample. From left to right, the plots are $20 \mathrm{deg}, 40 \mathrm{deg}$, and $60 \mathrm{deg}$, respectively.

The polished aluminum sample varies somewhat from the lab mirror sample and the data sets used to establish the baselines. Figure 5 shows the polished aluminum sample in direction cosine space. The sample appears to be stretched significantly in direction cosine space, showing clear asymmetry in this space in comparison to the lab mirror sample. Table 6 summarizes the results captured for the polished aluminum sample.

Referring to Table 6 , the polished aluminum has a higher standard deviation for all categories than the lab mirror sample. The diameter, even when scaled, has a standard deviation that is half of the mean. With the simulated isotropic sample, this standard deviation was $2 \%$ of the mean. Another significant difference was in the mean symmetry, where the percentage difference in symmetry (420.99\% versus $9.39 \%$ ) was nearly 45 times larger. This is likely due to the fact that there was stronger directional skewing in the polished aluminum, which is evidenced by the high standard deviation of eccentricity. Another point favoring anisotropy of this material is the standard deviations of the $x$ and $y$ centers, which are both at least six times that of the baseline standard deviation. Thus, the polished aluminum sample rates as more anisotropic than the lab mirror sample. This result matched predictions, as the visible machine lines on the surface caused one to theorize that this would create anisotropic reflection.

Table 6 The polished aluminum sample is rated as more anisotropic than the lab mirror sample. This is because for all categories, the standard deviation is significantly higher. The standard deviation for the diameter is half that of the mean, which is significant, compared to the standard deviation that was only $2 \%$ of the mean for the simulated isotropic material. The $(x, y)$ center (of every contour, not just the inner and outer contours for each $\theta_{i}$ ) shifts around much more, and the eccentricity has a high standard deviation, indicating strong directional skewing (in $x$ ).

\begin{tabular}{|c|c|c|c|c|c|c|c|}
\hline Material & $\begin{array}{l}\text { Mean } \\
\text { symmetry } \\
\text { (\% dif) }\end{array}$ & $\begin{array}{l}\text { Scaled } \\
\text { diameter } \\
\text { (pixels) }\end{array}$ & $\begin{array}{l}\text { Scaled } \\
\text { FWHM } \\
\text { (unitless) }\end{array}$ & $\begin{array}{c}X \text { center } \\
\text { (pixels) }\end{array}$ & $\begin{array}{l}Y \text { center } \\
\text { (pixels) }\end{array}$ & Ecc. & Class \\
\hline $\begin{array}{l}\text { Polished } \\
\text { aluminum }\end{array}$ & $\begin{array}{c}420.99 \\
\pm 195.63\end{array}$ & $\begin{array}{l}112.70 \\
\pm 55.64\end{array}$ & $\begin{array}{c}0.022 \\
\pm 0.006\end{array}$ & $\begin{array}{r}134.85 \\
\pm 48.89\end{array}$ & $\begin{array}{l}119.97 \\
\pm 20.13\end{array}$ & $\begin{array}{c}0.46 \\
\pm 0.19\end{array}$ & Anisotropic \\
\hline
\end{tabular}


Table 7 Table summarizing the initial prediction compared to the classification done by the algorithm.

\begin{tabular}{lccc}
\hline \hline Material & Prediction & Score & Class \\
\hline Lab mirror & Isotropic & 0.36 & Isotropic \\
Kapton & Leans isotropic & 10.38 & Anisotropic \\
Polished aluminum & Anisotropic & 13.25 & Anisotropic \\
Solar panel & Highly anisotropic & 42.59 & Highly anisotropic \\
Metal mesh & Anisotropic & $\mathrm{n} / \mathrm{a}$ & $\mathrm{n} / \mathrm{a}$ \\
\hline \hline
\end{tabular}

\subsection{Analysis and Summary of Results}

While the polished aluminum and lab mirror samples were evaluated in greater detail in this paper, the remaining samples (kapton, solar panel, and metal mesh) were also evaluated using the algorithm detailed in Sec. 3.1. The data and plots for these samples are omitted for brevity. The results of this algorithm are detailed in Table 7.

Overall, the algorithm was able to successfully identify four out of five materials as being conclusively isotropic or anisotropic. The conditions for which the algorithm will work were also defined, as samples where the BRDF peak drops below $100 \mathrm{sr}^{-1}$ and where (if modeled using the Beckmann distribution) $\sigma_{g}$ is $>0.1$ cannot be processed by this algorithm. The metal mesh sample was an example where the BRDF was too low, and the presence of the $2 \mathrm{D}$ diffraction grid broke the algorithm. The algorithm works best on highly specular materials and becomes less reliable as the material becomes more diffuse. The $(x, y)$ center and mean eccentricity criteria are the most robust parameters of the algorithm, able to measure rotational symmetry when the contour levels shift with $\theta_{i}$ better than the scaled diameter metric. Metrics that were flawed or need to be adjusted were the scaled diameter metric, which should be discarded, or adjusted to account for the alignment uncertainty, and the standard deviation of eccentricity, which should be adjusted to be the average standard deviation for each $\theta_{i}$. Overall, the lab mirror was the most isotropic and similar to the beam signature and simulated data. The solar panel was the most anisotropic material, as it produced two highly asymmetric peaks as well as a diffraction pattern, contributing highly to the anisotropicity. The Kapton and polished aluminum samples fall in between the lab mirror and solar panel in terms of anisotropicity. The Kapton sample, which was predicted to lean isotropic, measured barely anisotropic (just above 10). This is possibly due to the fact that if Kapton is not laid completely flat, the directional reflectance can change. As stated previously, the final results are summarized in Table 7.

\section{Conclusion}

The developed algorithm was able to identify materials of varying isotropy, working best on highly specular materials. Each of the materials was first classified as isotropic or anisotropic by a qualitative assessment of the material's surface. The first sample was the lab mirror which was the smoothest appearing surface (and was qualitatively classified as such), and the algorithm was able to identify it as being slightly less isotropic than the beam signature, which was the baseline. The second sample was a Kapton film, which is a semi-transparent film, with no visible machine lining, and some scratches. The algorithm classified it as semi-anisotropic, which is likely due to the sample not being laid completely flat, changing the directional reflectance. The next sample was the polished aluminum, which was more clearly anisotropic as the circular machining lines on the sample were readily visible. As such, the algorithm classified this surface as more anisotropic than the lab mirror sample. The fourth material was a solar panel sample, which consisted of a top clear layer, and a darker surface underneath with a series of horizontal grid conduction lines. The sample is clearly anisotropic due to the directionality of the grid lines (which generated a diffraction pattern). The algorithm was able to successfully identify this 
surface as anisotropic. The final sample was the metal mesh, which was unable to be classified. However, this sample was useful in determining the limits of the algorithm. Ultimately, it was found using simulated data, when the BRDF peak falls below $100 \mathrm{sr}^{-1}$, samples cannnot be classified by the algorithm. Thus, samples with $\sigma_{g}>0.1$ (using the isotropic Beckmann distribution function) are too optically rough to be classified.

Overall, the algorithm is robust for relatively specular materials, as it employs multiple parameters to successfully analyze out-of-plane BRDF data to classify the degree of isotropy of materials. Due to its ability to classify materials depending on their degree of isotropy, this algorithm will improve modeling of optical scatter. For example, in light curve analysis, understanding that a solar panel will provide an anisotropic reflection helps not only with tracking of space-based objects, but also in determining other characteristics of said object such as its geometry. Remote sensing and scene generation applications also rely upon accurate BRDF models. For example, in remote sensing, accurate modeling of BRDF allows users to determine properties of a scene. This algorithm is better able to classify materials than attempting to fit BRDF data to existing models and then determining the degree of isotropy. By circumventing the step of fitting the data to models to determine the surface characteristics, the BRDF can be more accurately characterized, improving the process of extracting scene properties for remote sensing.

For future work, improvements to the robustness of some of the metrics in the algorithm could be made, as some were more reliable indicators of isotropy than others. For example, the mean symmetry metric was better at identifying material isotropy than the scaled diameter metric. The algorithm could also be extended to determine the degree of isotropy present in more diffuse materials. Another possible study could be to test the algorithm over a range of wavelengths. Since materials become more specular at longer wavelengths and begin to exhibit directional reflection, any accompanying anisotropy which develops spectrally could be studied.

\section{Acknowledgments}

The views expressed in this paper are those of the authors and do not necessarily reflect the official policy or position of the United States Air Force, the U.S. Department of Defense or the U.S. Government.

\section{References}

1. F. Nicodemus et al., "Geometrical considerations and nomenclature for reflectance," National Bureau of Standards Monograph 160 (1977).

2. J. T. Kajiya, "The rendering equation," ACM SIGGRAPH 20(4), 143-150 (1986).

3. A. Ceniceros et al., "Comparison of BRDF-predicted and observed light curves of GEO satellites," in Proc. Adv. Maui Opt. and Space Surv. Technol. (2015).

4. K. Torrance and E. Sparrow, "Theory of off-specular reflection from roughened surfaces," J. Opt. Soc. Am. 57, 1105-1114 (1967).

5. S. Rusinkiewicz, "A new change of variables for efficient BRDF representation," in Proc. Eurogr. Workshop Rendering (1998).

6. S. Butler and M. Marciniak, "Robust categorization of microfacet BRDF models to enable flexible application-specific BRDF adaptation," Proc. SPIE 9205, 920506 (2014).

7. B. Ewing, S. Butler, and M. Marciniak, "Improved grazing angle bidirectional reflectance distribution function model using Rayleigh-Rice polarization factor and adaptive microfacet distribution function," Opt. Eng. 57(10), 105102 (2018).

8. G. J. Ward, "Measuring and modeling anisotropic reflection," ACM SIGGRAPH Comput. Graphics 26(2), 265 (1992).

9. R. Cook and K. Torrance, "A reflectance model for computer graphics," ACM Trans. Graph. 1(1), 7-24 (1982).

10. R. Priest and T. Germer, "Polarimetric BRDF in the microfacet model," in Proc. Meet. MSS Speciality Sens. Group Passive Sens., Vol. 1, pp. 169-182 (2000).

11. D. Wellems et al., "Long wave infrared polarimetric model theory, measurements and parameters," J. Opt. A 8, 914-925 (2006). 
12. J. Blinn, "Models of light reflection for computer synthesized pictures," in Proc. 4th Annu. Conf. Comput. Graphics and Interactive Tech. (1977).

13. M. Ashikhman and P. Shirley, "An anisotropic phong BRDF model," J. Graph. Tools 5(2), 25-32 (2000).

14. R. Montes and C. Urena, "An overview of BRDF models," Technical Report LSI-2012-001, University of Grenada (2012).

15. J. Harvey, A. Krywonos, and C. Vernold, "Modified Beckmann-Kirchhoff scattering model for rough surfaces with large incident and scattering angles," Opt. Eng. 46, 078002 (2007).

16. S. Butler and M. Marciniak, "Comparison of microfacet BRDF model to modified Beckmann-Kirchhoff BRDF model for rough and smooth surfaces," Opt. Express 23(22), 29100 (2015).

17. T. V. Small, S. D. Butler, and M. A. Marciniak, "Augmenting CASI ${ }^{\circledR}$ BRDF measurement device to measure out-of-plane scatter with CCD pixel array," Proc. SPIE 11485, 114850B (2020).

18. T. V. Small, S. D. Butler, and M. A. Marciniak, "Scatter coordinate mapping and out-ofplane BRDF measurements for specular materials using an augmented CASI ${ }^{\circledR}$ measurement system," Proc. SPIE 11727, 117270X (2021).

19. T. V. Small, S. D. Butler, and M. A. Marciniak, "Uncertainty analysis for CCD-augmented CASI BRDF system," Manuscript submitted for publication (2021).

Anne W. Werkley is currently a Captain in the United States Air Force. She obtained her bachelor's degree in physics from the United States Air Force Academy in 2017, and her master's degree in applied physics from the Air Force Institute of Technology (AFIT) in 2021. She has previously served at Kirtland Air Force Base, New Mexico, and currently works at the Air Force Research Laboratory, Wright-Patterson AFB, Ohio.

Samuel D. Butler is a Lieutenant Colonel in the United States Air Force and an assistant professor at the AFIT. He received his BS degree in applied physics with a computer science emphasis from Brigham Young University, his MS degree in applied physics, and his PhD from AFIT. His research interests include bidirectional scatter. He has advised seven MS and $\mathrm{PhD}$ students, and published 20 technical papers. He is a member of SPIE.

Todd V. Small: Biography is not available.

Michael A. Marciniak is a professor at the AFIT. He received his BS degree in mathematics from St. Joseph's College, India, BSEE degree from the University of Missouri-Columbia, and MSEE and PhD degrees from AFIT. His research interests include bidirectional scatter distribution and scatterometry of optical metasurfaces. He has advised $12 \mathrm{PhD}$ dissertations and 55 MS theses, and published 41 refereed and 80 technical papers. He is a senior member of SPIE. 